\title{
A Theoretical Investigation of the Effect of Latitude on Avian Life Histories
}

\author{
John M. McNamara, ${ }^{1, *}$ Zoltán Barta, ${ }^{1,2,3, \dagger}$ Martin Wikelski, ${ }^{4, \star}$ and Alasdair I. Houston ${ }^{3, \$}$
}

1. Department of Mathematics, University of Bristol, University Walk, Bristol BS8 1TW, United Kingdom;

2. Department of Evolutionary Zoology, University of Debrecen, Debrecen Egyetem tér 1., 4032, Hungary;

3. School of Biological Sciences, University of Bristol, Woodland Road, Bristol BS8 1UG, United Kingdom;

4. Department of Ecology and Evolutionary Biology, Princeton University, Princeton, New Jersey 08544; and Max Planck Institute for Ornithology, Schlossallee 2, 78315 Radolfzell, Germany

Submitted April 2, 2007; Accepted February 19, 2008;

Electronically published July 30, 2008

Online enhancements: appendixes.

Aвstract: Tropical birds lay smaller clutches than birds breeding in temperate regions and care for their young for longer. We develop a model in which birds choose when and how often to breed and their clutch size, depending on their foraging ability and the food availability. The food supply is density dependent. Seasonal environments necessarily have a high food peak in summer; in winter, food levels drop below those characteristic of constant environments. A bird that cannot balance its energy needs during a week dies of starvation. If adult predation is negligible, birds in low seasonal environments are constrained by low food during breeding seasons, whereas birds in high seasonal environments die during the winter. Low food seasonality selects for small clutch sizes, long parental care times, greater age at first breeding, and high juvenile survival. The inclusion of adult predation has no major effect on any life-history variables. However, increased nest predation reduces clutch size. The same trends with seasonality are also found in a version of the model that includes a condition variable. Our results show that seasonal changes in food supply are sufficient to explain the observed trends in clutch size, care times, and age at first breeding.

Keywords: annual routine, clutch size, energy balance, parental care.

\footnotetext{
* E-mail: john.mcnamara@bristol.ac.uk.

† E-mail: zbarta@delfin.unideb.hu.

¥ E-mail: martin@orn.mpg.de.

§ E-mail: a.i.houston@bristol.ac.uk.
}

Am. Nat. 2008. Vol. 172, pp. 331-345. (c) 2008 by The University of Chicago. 0003-0147/2008/17203-42512\$15.00. All rights reserved.

DOI: $10.1086 / 589886$
The pioneering work of Moreau (1944) and Lack (1947) suggested that the clutch size of birds increases with latitude; that is, birds that breed near the equator tend to have smaller clutches than birds that breed away from the equator. Subsequent work has provided a rigorous justification of this trend by using two ways to demonstrate the effects of latitude. One way is to investigate the lifehistory parameters for a species that breeds at a range of latitudes. This method has been applied to the tree swallow Tachycineta bicolor (Dunn et al. 2000), the great tit Parus major (Sanz 1998), the flicker Colaptes auratus (Koenig 1984), the house wren Troglodytes aedon (Young 1994), and the stonechat Saxicola torquata (König and Gwinner 1995). As an example, Dunn et al. (2000) found a significant increase in the clutch size of tree swallows with breeding latitude in North America. The second method is to carry out a comparative analysis (e.g., Böhning-Gaese et al. 2000; Martin et al. 2000; Cardillo 2002; Martin 2002). Böhning-Gaese et al. (2000) analyzed the clutch size of 373 species of North American birds and 252 species of European birds and found a significant increase in clutch size with latitude. The number of clutches per year decreased with latitude.

There is evidence that the clutch size pattern is linked to environmental parameters, such as the seasonal food flush, or seasonality of migrants (Ricklefs 1980; Lepage and Lloyd 2004). Further research has revealed that several other aspects of avian life history also depend on latitude (Ricklefs 2000a; Martin et al. 2006). Birds that breed near the equator ("tropical birds") care for their young for a long time (Russell 2000; Schaefer et al. 2004; Styrsky et al. 2005). Young tropical birds tend to have slow growth rates (Ricklefs 1976; Bryant and Hails 1983), they do not become mature as soon as the young of nontropical species (Skutch 1976; Russell 2000; Russell et al. 2004), and senescence is delayed (Møller 2007). The view that tropical birds tend to have high survival as adults was challenged by Karr et al. (1990), who found no difference in the survival of tropical and temperate forest birds, but there 
are problems with this conclusion (Johnston et al. 1997; Sandercock et al. 2000; Stutchbury and Morton 2001). The comparative analysis presented by Peach et al. (2001) shows that the survival rate of southern African songbirds is higher than that of European songbirds. Although more work is needed on this topic, there is evidence in support of higher adult survival in the tropics.

Ultimate selection pressures influencing the above-named traits may also have been instrumental in shaping widespread differences in metabolic rate between temperate-zone and tropical birds (Ricklefs 1976; Weathers 1979, 1997; Klaassen and Drent 1991; Klaassen 1994; Tieleman et al. 2005). In general, tropical birds have lower metabolic rates, that is, a slower "pace of life" (Wikelski et al. 2003; Wiersma et al. 2007). Metabolic rate is often seen as the major hub in the network of physiological mechanisms underlying lifehistory traits and is potentially a heritable trait (Wikelski et al. 2003). Differences in metabolic rate are expected to mediate some or all of the major life-history trade-offs mentioned above (Ricklefs and Wikelski 2002).

Understanding the relative influence of the proposed ultimate reasons underlying latitudinal life-history gradients is a major challenge (Cody 1966; Martin 1996; Cardillo 2002). Various explanations have been suggested, the most popular being based on differences in food and predation, both of which could be mediated by differences in day length and temperature. One of the more widely accepted explanations to date for latitudinal clutch size variation has been Ashmole's hypothesis, which states that clutch size is higher at higher latitudes because of the seasonal flush of resources resulting from fewer competing consumers in more-seasonal environments (Ashmole 1963). As one travels toward the poles, annual variations in day length, incident solar radiation, and primary productivity grow more extreme. On this view, what is important is not the maximum level of resources over the year but the maximum level relative to the minimum level. Ashmole's (1963) ideas were elaborated and strengthened by Ricklefs (1980), who estimated the relative availability of food by the ratio between primary plant production in summer and that in winter. Using such a measure, researchers found significant positive correlations between the relative summer productivity and clutch size in woodpeckers (Koenig 1984, 1986) and various passerines (Ricklefs 1980), including tree swallows (Dunn et al. 2000).

An alternative explanation (not exclusive to the one above) is that high nest predation rates in the tropics are ultimately responsible for small clutch sizes. It has been suggested that high nest predation could select for multiple renesting attempts, short development time, and small eggs and clutches (Martin 1996). However, high nest predation could also result in slower development rates because adults might reduce their nest attentiveness and parental feeding visits, resulting in food limitation (Ghalambor and Martin 2001). The empirical evidence for the influence of nest predation on avian life-history traits is mixed. Robinson et al. (2000) found high interannual variation in nest predation rate in tropical Panama, as well as extensive overlap of the absolute levels of nest predation between tropical and temperate-zone habitats (see also Martin et al. 2006). Another study comparing ecologically and phylogenetically similar species in North America and subtropical South America suggested that nest predation may explain clutch size differences within, but not between, latitudes (Martin et al. 2000). Other possible explanations are based on ambient temperature (e.g., Reid et al. 2000; Cooper et al. 2005) and the consequences of microbial infection for embryo mortality before the onset of full incubation (Cook et al. 2003, 2005; Beissinger et al. 2005).

In summary, it appears that there exist too many potential selective influences on clutch size and other lifehistory traits to explore them with verbal arguments alone. We suggest that the time is ripe for developing an analytical model that includes most of the factors mentioned above and using it to establish how these factors could influence the life histories of tropical and temperate-zone birds. We believe that previous accounts of the effect of latitude on life-history evolution in birds are limited for two related reasons. If population size is stable, then there are constraints on life-history parameters (e.g., Ricklefs $1977 a$, 1977b; Charnov 1986, 1993; Sutherland et al. 1986). Although Lack (1947) and Ashmole (1963) discussed such constraints, they were not included in a formal model of latitude, and hence their full implications were not perceived. When the previous accounts were being developed, it was not possible to construct appropriate models because there was no adequate theoretical basis. An adequate model must consider how the seasonality of the environment influences optimal behavior over the entire year. This requires a model of optimal annual routines. Houston and McNamara (1999; see also McNamara and Houston 2008) describe a powerful general framework for finding such routines.

In this article, we provide the first explicit and selfconsistent account of a latitudinal trend in a suite of lifehistory parameters. It is not feasible to explore all possible explanations; instead, we concentrate on whether trends can be explained by Ashmole's (1963) hypothesis. Our aim in this article is to show that the interaction between seasonality of food and density dependence is sufficient to explain many of the differences that have been observed between tropical and temperate bird species. We are not claiming that no other factors operate, nor are we claiming that latitudinal trends will always exist. What we are saying 


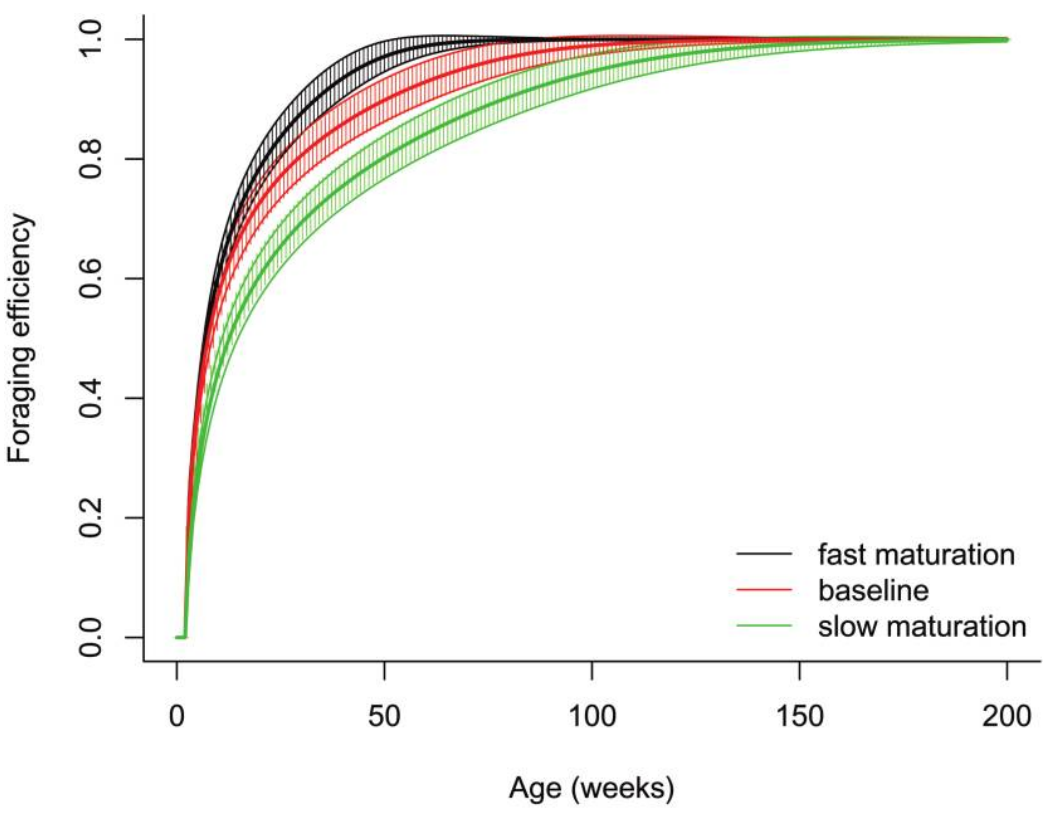

Figure 1: Mean and standard deviation in foraging efficiency as a function of age. The three cases shown are, from top to bottom, fast maturation, baseline, and slow maturation. Details of functions are given in appendix A in the online edition of the American Naturalist.

is that if all else is equal, then the seasonality of food is a sufficient explanation.

We predict breeding phenology by analyzing the optimal scheduling of reproductive activities over the annual cycle, using a model of a schematic bird. In this model, the food supply varies over the year but is the same at a given time and latitude in successive years. Thus, there is no year-toyear variation. The food supply is density dependent, and to check the robustness of our results, we consider more than one form of density dependence. We are primarily concerned with the effects of the constraint imposed by the food supply when there is no predation and how results are modified when there is a trade-off between obtaining food and avoiding predation.

\section{The Model \\ Decisions}

There are 52 decision epochs per year (weekly decisions). A bird at the start of week $t$ (time $t$ ) has the following options. If the bird has no brood, it can (a) delay-that is, not start a brood-or $(b)$ start-that is, lay a clutch of eggs. If it decides to start a brood, it must choose its clutch size, $n$ (a positive integer). Production of the brood takes one time unit, so the eggs appear at time $t+1$. If the bird has a brood, it can (a) keep or (b) abandon the brood. This takes place instantaneously at time $t$. The decision taken and the food supply determine the amount of time during the week that is spent foraging (see below). This time, $u$, is thus an outcome of the reproductive decisions.

\section{Food Availability}

Time of year $t=0$ is midwinter, $t=26$ is midsummer, and $t=52$ is midwinter and time $t=0$ of the following year. Food availability at time $t$ is

$$
g(t)=A\left[1+\varepsilon_{\mathrm{F}} \sin \left(\frac{t-13}{26} \pi\right)\right]
$$

Here the parameter $\varepsilon_{\mathrm{F}}$ characterizes the degree of seasonality in the food supply. The parameter $A$ is a measure of the overall food availability in the environment. In the basic model, we assume that density dependence acts through the constant $A$; specifically, $A$ decreases as average annual population size increases. To investigate the robustness of results to the form of density dependence assumed, we also consider an alternative form in appendix $\mathrm{D}$ in the online edition of the American Naturalist.

\section{Efficiency and Energy Intake}

The ability of a bird to obtain food tends to increase as it gets older, that is, as it matures (e.g., Weathers and Sullivan 1989). Specifically, a bird's foraging efficiency $\theta$ increases from $\theta=0$ at birth to its maximum value 
$\theta=1$. The details of this increase are given in appendix $\mathrm{A}$ in the online edition of the American Naturalist. We present results for three scenarios, which we refer to as "slow maturation," "baseline," and "fast maturation" (fig. 1). We explicitly assume that the parent does not know the exact efficiency of its offspring during care or at abandonment, although the longer the care period, the greater the mean efficiency. A bird with efficiency $\theta$ that forages for a time $u$ in week $t$ finds food with energetic content $\theta u g(t)$.

\section{Energy Expenditure and Balance}

We assume that the energy expenditure over a week equals the energy intake. This seems a reasonable assumption for a small bird, although balance over a single week would be a poor approximation for a large bird.

The resting metabolic expenditure in week $t$ is $c(t)$. In our baseline case, we assume thermoneutrality and that one unit of energy is used while resting at all times of year $(c(t)=1$ for all $t)$. The rate of expenditure increases by $m$ while a bird is searching for food. Thus, a bird that delays breeding expends energy $c(t)+m u$ during a week.

A bird with maximum efficiency that starts a clutch expends energy $\Delta_{\text {nest }}$ on building the nest and expends energy $\Delta_{\text {off }}$ on each egg produced. Its total expenditure if it produces $n$ eggs is thus $c(t)+m u+\Delta_{\text {nest }}+n \Delta_{\text {off }}$. During care, it supplies each offspring with food of energy content $F_{\text {off }}$ each week. Its rate of finding food while searching is the same regardless of whether it is finding food for itself or its offspring. We assume, however, that the bird is a central-place forager (Orians and Pearson 1979) while feeding young, transporting food found at the foraging area to the nest at rate $D$. Thus, it takes total time $u_{\mathrm{Y}}=n F_{\text {off }} / D$ to transport the required amount of food to a clutch of size $n$. During transport, metabolic rate is increased above the resting rate by $m_{\mathrm{fly}}$. Overall weekly expenditure is thus $c(t)+m u+m_{\mathrm{fly}} u_{\mathrm{Y}}$. Details of how $u$ and $u_{\mathrm{Y}}$ can be found are given in appendix $\mathrm{B}$ in the online edition of the American Naturalist.

\section{Efficiency and Reproduction}

We have no state variable representing physiological maturity in our model, so that even a newly independent offspring is physiologically capable of reproduction. However, the low foraging efficiency of a young bird (see above) makes it harder for it to obtain food for itself and any young. Since immature birds are liable to be poorer at other aspects of reproduction, we additionally assume that an immature bird with efficiency $\theta$ expends energy $\Delta_{\text {nest }} / \theta^{J}$ on building the nest and that during transport of food to the young, it increases its metabolic rate above the resting rate by $m_{\mathrm{fly}} / \theta^{J}$. We refer to $J$ as the juvenile disadvantage parameter. In the baseline case, $J=2$. However, as we demonstrate in "Robustness of the Results," most results are highly robust to the choice of this parameter.

\section{Sources of Mortality}

(i) If a bird cannot balance its energy expenditure and energy gain during a week, it dies of starvation. We envisage that, because of uncertainties in the weather and other disturbances, the total time available to forage during a week is a random variable. We take our time unit so that this random variable has mean 1 . Motivated by this, we consider a bird that requires time $u_{\text {tot }}$ to obtain sufficient food (in the case of a single bird, $u_{\text {tot }}=u$; if caring for young, $u_{\mathrm{tot}}=u+u_{\mathrm{Y}}$ ). Then the bird manages to balance its energy needs with probability $1 /\left(1+u_{\mathrm{tot}}^{K}\right)$. Here the parameter $K$ is large, so that the probability of energy balance is almost a step function that drops from 1 to 0 at $u_{\mathrm{tot}}=1$. Note that a bird with young always has the chance to reduce the probability that it will starve by abandoning the young. (ii) Death from predation occurs during a week with probability $\mu u^{2}$. (iii) Mortality from all other sources occurs with constant probability $\varphi$ during a week.

\section{Offspring Mortality}

If the young are abandoned by the parent at time $t$, they become independent immediately (i.e., at $t$ ). If the parent does not abandon at $t$ but dies between times $t$ and $t+$ 1 , all young become independent at time $t+1$. If the parent does not abandon at $t$ and survives until time $t+1$, then the nest is predated between $t$ and $t+1$ and all young die, with probability $\mu_{\text {off }}$.

\section{Evolutionary Stability}

A strategy is a rule specifying how a bird's choice of action depends on the bird's efficiency, reproductive status (i.e., whether there are dependent young and the age and the number of any dependent young), and time of year. Consider an environment with given food availability over the year, as specified by the function $g$. Within this environment, a strategy has an associated annual projection matrix. The fitness of the strategy is the maximum eigenvalue of this projection matrix (Caswell 2001). This is the asymptotic annual proportional growth rate in numbers following the strategy within the fixed environment. The strategy maximizing fitness within this environment can be found by dynamic programming (McNamara 1991; Houston and McNamara 1999; Clark and Mangel 2000).

Following Metz et al. (1992), we seek an evolutionarily 
Table 1: Definition of parameters

\begin{tabular}{llc}
\hline Parameter & \multicolumn{1}{c}{ Interpretation } & Baseline value \\
\hline$\varepsilon_{\mathrm{F}}$ & Seasonality in the food supply & 1.0 \\
$c(t)$ & Weekly resting metabolic expenditure & 5.0 \\
$u$ & Proportion of available time (daylight) in the week spent foraging & 7.5 \\
$m$ & Additional metabolic expenditure per unit time foraging & .2 \\
$m_{\text {fly }}$ & Additional metabolic expenditure per unit time transporting food to the young & .8 \\
$\Delta_{\text {off }}$ & Expenditure per egg produced & 12.5 \\
$\Delta_{\text {nest }}$ & Expenditure on nest construction & .5 \\
$D$ & Rate of transport of energy in food from the foraging area to the nest & $10^{-6}$ (energy constraint), \\
$F_{\text {off }}$ & Weekly energy requirement of each offspring & .01 (predation risk) \\
$\mu$ & Parental predation risk parameter & .0025 (energy constraint), \\
& Weekly background mortality risk to parent & .0005 (predation risk) \\
$\varphi$ & Weekly predation risk to young & 0 \\
$\mu_{\text {off }}$ & Starvation parameter & 200 (energy constraint), \\
$K$ & Juvenile disadvantage factor for reproduction by inexperienced birds & 40 (predation risk) \\
$J$ & & 2.0 \\
\hline
\end{tabular}

stable strategy (ESS). That is, we seek a strategy such that if almost all population members adopt this strategy, then no rare mutant within this population has higher fitness.

To find this strategy when density dependence acts through the parameter $A$ (eq. [1]), we adjust $A$ during computation so that the maximum fitness equals 1 . Then, as is shown in appendix D, the strategy that maximizes fitness for this $A$ is the unique ESS. When density dependence has more complex forms, a more computationally intensive procedure is required (app. D).

\section{Results}

\section{Results in the Energy-Constraint Case}

We first consider what we refer to as the "energy-constraint" version of our model. In this version, we assume that there is no nest predation. Birds are thermoneutral, so that seasonality is solely the result of seasonal changes in the available food $(c(t)=1$ for all $t)$. Predation risk is almost negligible $\left(\mu=10^{-6}\right)$; it is not 0 so as to avoid degenerate cases in which breeding and not breeding are both optimal. The survival function $1 /\left(1+u_{\text {tot }}^{K}\right)$ is very steplike at $u_{\text {tot }}=1(K=200)$. The survival function is not an exact step function because in that case, fitness would be a discontinuous function of the environmental food parameter $A$ and there may be no ESS.

In this version, there is essentially no penalty for high foraging intensity, provided that the bird's food requirement does not compel it to forage for close to the whole week. Consequently, a bird will always breed if doing so is energetically possible and provides any small advantage. This version therefore allows us to investigate the effects of the constraint imposed by the food supply.
Results are first presented for five cases of the energyconstraint version. The first case has baseline maturation (fig. 1) and baseline parameters (table 1). Two cases differ from this baseline in terms of the food requirements of dependent young. These cases are labeled "low need" $\left(F_{\text {off }}=0.25\right)$ and "high need" $\left(F_{\text {off }}=1.0\right)$. The cases labeled "fast maturation" and "slow maturation" differ from the baseline case in the rate at which young gain foraging efficiency (fig. 1).

Effects of the Constraint on Energy Intake Imposed by Density Dependence. The mean food in the environment (as measured by the parameter $A$ ) adjusts with $\varepsilon_{\mathrm{F}}$ so that the

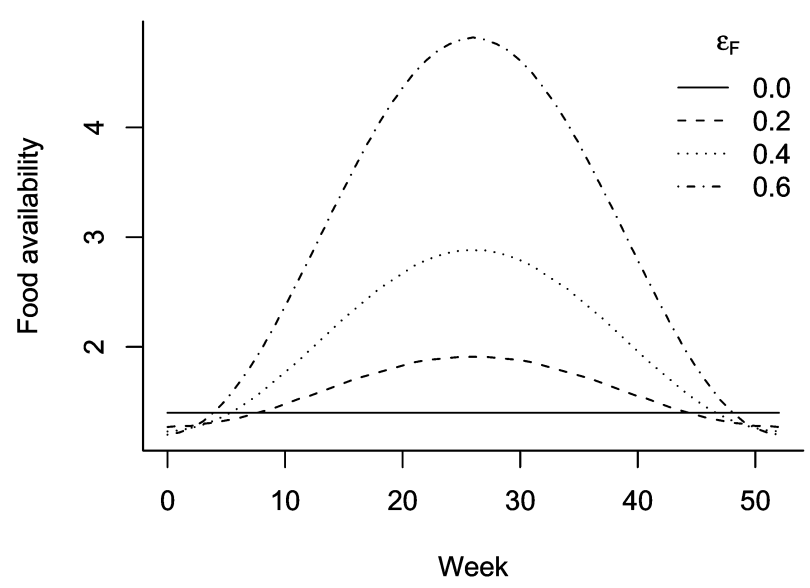

Figure 2: Food availability $g(t)$ (eq. [1]) at evolutionary stability as a function of time of year $t$ for various values of the seasonality in the food supply $\varepsilon_{\mathrm{F}}$. Energy-constraint baseline case. 
population has stable density at the ESS. An adjustment that improved (reduced) food at all times of year would necessarily increase (decrease) population growth rate, so that the population density would not be stable. It follows that as $\varepsilon_{\mathrm{F}}$ increases, midwinter food must decrease and midsummer food must increase. Figure 2 illustrates the change in food availability as seasonality changes. As figures 2 and $3 A$ show, as $\varepsilon_{\mathrm{F}}$ increases from 0 to 0.6 , midwinter food availability typically decreases by around $10 \%$. Given function (1), this implies that the increase in midsummer food availability is typically more than $300 \%$. In other words, the winters get a little worse, but the summers get a lot better.

For low $\varepsilon_{\mathrm{F}}$, the annual bottleneck is during breeding (which may be in winter; fig. 3). For higher $\varepsilon_{\mathrm{F}}$, breeding occurs in spring or summer, and the bottleneck is for nonbreeding birds in winter.

For low $\varepsilon_{\mathrm{F}}$, the breeding bottleneck is greatest in the high-need case, which has the greatest cost of care of one offspring, so this case has the highest midwinter food availability and hence the smallest equilibrium winter population density. For high $\varepsilon_{\mathrm{F}}$, a parent can easily care for at least one offspring, so the high-need case no longer has the greatest midwinter food availability (fig. $3 A$ ). At high $\varepsilon_{\mathrm{F}}$, the major limiting factor is survival of young over winter, so the slow-maturation case has the highest midwinter food availability (fig. 3A).

Mean Care Times and the Number of Clutches per Year. Consider first the case $\varepsilon_{\mathrm{F}}=0$. In this case, after young from one breeding attempt become independent, there is no possible advantage to delay, and it is optimal to breed again after the minimum possible delay (1 week). Thus, in this case, care times plus 1 week and mean numbers of clutches per year are inversely proportional (see fig. $3 B$, $3 C$ ). Care times are mainly determined by the time needed for newly independent young to have sufficient foraging efficiency to survive (fig. $3 B$ ). Thus, care times are long when efficiency increases slowly (slow-maturation case) and short when gain is rapid (fast-maturation case). Because food is better when breeding is hard (high-need case), care times are also shorter in that case. Note that this shorter care time arises without an assumption that high need means faster development.

As $\varepsilon_{\mathrm{F}}$ increases, the care period becomes constrained because there is not enough food to supply even one offspring in winter. All care must now fit into a seasonal breeding window. Thus, the first clutch is always laid at the start of the breeding window, and reproduction becomes locked into the annual cycle. We refer to the phenomena of locking into the annual cycle as entrainment (McNamara et al. 2004). For each case, there is a critical value of $\varepsilon_{\mathrm{F}}$ below which there is no entrainment and above which reproduction is entrained. The transition to entrainment occurs when the number of clutches first switches to 1 as $\varepsilon_{\mathrm{F}}$ increases (fig. $3 C$ ). Typically, entrainment sets in before $\varepsilon_{\mathrm{F}}=0.1$. The slow-maturationconstrained case is exceptional because the young gain foraging efficiency slowly and the optimal care time is still longer than a year when $\varepsilon_{\mathrm{F}}=0.1$.

As $\varepsilon_{\mathrm{F}}$ increases, there is more food available in summer. Thus, young are able to care for themselves at a younger age. Care times tend to decrease, and it becomes possible to fit more than one clutch into the breeding window (fig. $3 B, 3 C)$. The number of clutches per year increases monotonically with increasing $\varepsilon_{\mathrm{F}}$ in all cases (fig. $3 C$ ).

There is more food overall as $\varepsilon_{\mathrm{F}}$ increases and the summer becomes much better. Thus, the duration of the breeding window increases as $\varepsilon_{\mathrm{F}}$ increases. This leads to longer care periods for ranges of $\varepsilon_{\mathrm{F}}$ over which clutch number per year is constant in the constrained cases. The increase in the length of the breeding window and the increase in the number of clutches produced result in alternating slow increases and abrupt drops in the mean care period as $\varepsilon_{\mathrm{F}}$ increases (fig. 3B).

Lay Date. The lay date of the first clutch to become independent in a year is shown in figure $3 D$. For the low$\varepsilon_{\mathrm{F}}$, low-need case, it is optimal to lay in the previous year and care over winter. This ensures that young become independent during spring, allowing them to gain experience before winter. This strategy applies particularly when young gain foraging efficiency slowly (slow maturation) or when they gain it at a medium rate but require less food (low need). For higher $\varepsilon_{\mathrm{F}}$, as $\varepsilon_{\mathrm{F}}$ increases and food in summer increases, lay dates become earlier.

Clutch Size. As seasonality increases, the food in summer gets better, and it becomes possible to sustain a bigger clutch. Figure $3 E$ plots the mean number of eggs per clutch averaged over all clutches produced in a year. When there is more than one clutch, the first has fewer eggs than the second.

Year of First Breeding. Figure $3 F$ shows the year of first breeding. For low seasonality, there is a tendency to delay breeding until the second year after birth unless young gain foraging efficiency rapidly (fast-maturation case). As seasonality increases, there is more food in summer, and it becomes optimal to breed in the first year after birth. Finally, for high seasonality, it is optimal for young to breed in the year of their birth.

Juvenile Survival. Figure $3 G$ shows the survival of juveniles to the end of the first winter after their time of independence. For low seasonality, parental care lasts a year or 
more in some cases (fig. 3B). Not surprisingly, survival is highest in these cases because juveniles are already quite efficient foragers at independence.

As seasonality increases, all care times become shorter, so that young are less efficient at foraging at independence. This, combined with reduced food availability in midwinter, dramatically reduces the probability that juveniles survive their first winter. The decreased survival with increasing seasonality can also be seen as an inevitable consequence of the increased numbers of young produced and the fact that populations are at a density-dependent equilibrium. Increased seasonality increases competition between juveniles for food (as represented by the decreased midwinter food), and survival is reduced.

Adult Survival. Once reproduction is entrained, adult survival is almost independent of seasonality in four of the five cases shown in figure $3 \mathrm{H}$. In these four cases, there is little adult starvation, and survival probability is largely determined by the background mortality. In contrast, in the fast-maturation case, adult survival decreases strongly with increasing seasonality. This occurs because of the low food in midwinter. Another way to interpret this result is to note that juveniles are typically as good at foraging as their parents by their first winter. Thus, these individuals are in competition with older birds over food in midwinter, and there is high adult mortality as well as high juvenile mortality.

Robustness of the Results. In figure 3, we illustrated the effects of changing the food requirement of dependent young. There are four other parameters that control the energetic consequences of breeding in our model. Two concern initiation of breeding: $\Delta_{\text {nest }}$ is the metabolic expenditure required to build the nest, and $\Delta_{\text {off }}$ is the cost of egg production. Two concern delivery of food to the young: $m_{\mathrm{fl}}$ is the cost of transport of food, and $D$ is the rate of transport of food. We have investigated the robustness of our conclusions to changes in all four parameters. The effect of seasonality $\varepsilon_{\mathrm{F}}$ on all eight measures presented in figure 3 is highly robust regardless of the values of these breeding parameters (fig. $\mathrm{C} 1$ in the online edition of the American Naturalist). The effects of a change in a breeding parameter for given $\varepsilon_{\mathrm{F}}$ depend on details such as the number of clutches before the change (fig. C1). There are, however, some general trends: making breeding more difficult increases the food availability; making the initiation of breeding more difficult increases lay date when seasonality is low; making delivery of food to the young more difficult tends to reduce clutch size.

Reducing the background mortality $\varphi$ from its baseline value reduces overall food availability, increasing lay date and age of first breeding (fig. C2 in the online edition of the American Naturalist). The qualitative effect of increasing $\varepsilon_{\mathrm{F}}$ is unaltered.

In the results presented in figure 3, the juvenile disadvantage factor $J=2$. We have repeated calculations with a range of values of the juvenile disadvantage factor $J$ (fig. C2). The main effect of an increase in $J$ from $J=1$ (no disadvantage) to $J=8$ is a slight increase in the age of first breeding. However, the effect is surprisingly small; at high seasonality, even an eightfold disadvantage does not prevent juveniles from breeding in their year of birth. Increasing $J$ also tends to reduce the number of clutches and eggs per clutch at high seasonality. Other measures shown in figure 3 are highly robust to changes in $\varepsilon_{\mathrm{F}}$.

\section{Results from the Predation-Risk Version}

We now introduce a "predation-risk" version of our model. The baseline case of this version (table 1) is identical to the baseline energy-constraint case except that (i) background mortality is reduced, (ii) foraging incurs a predation risk, and (iii) foraging is more stochastic $(K=40)$. We introduce more stochasticity because, in contrast to the energy-constraint case, we want the birds to be able to control their probability of starvation (see McNamara and Houston 1987; Houston and McNamara 1993). By comparing predictions of the two versions, we are able to understand what effects are primarily a consequence of energy constraints and what additional effects are the result of a trade-off between mortality (starvation and predation) and reproduction.

The effect of changing from the energy-constraint baseline case shown in figure 3 to the corresponding predationrisk baseline case is shown in figure 4 . The effects of changing from each of the other four energy-constraint cases shown in figure 3 to the corresponding predation-risk cases are shown in figure C3 in the online edition of the American Naturalist. In all cases, the qualitative effects of food seasonality $\varepsilon_{\mathrm{F}}$ on each of the eight measures shown is unaltered by the change of version. The effects of changes in maturation speed and food need of dependent young are also highly robust.

In the predation-risk version, predation risk increases with foraging intensity. This tends to result in lower reproductive effort in the predation-risk cases than in the energy-constraint cases. This has several consequences. Entrainment tends to set in for smaller $\varepsilon_{\mathrm{F}}$ because, even if it is possible, breeding in midwinter requires high foraging intensity. In the predation cases, birds are usually not up against the energy constraint when they breed, and care times tend to be shorter; consequently, mean care times essentially decrease monotonically with increasing $\boldsymbol{\varepsilon}_{\mathrm{F}}$. The number of eggs per clutch is substantially reduced 
- baseline

$\boxminus$ low need

$\square$ high need

$\square$ fast maturation $\quad \nabla$ slow maturation
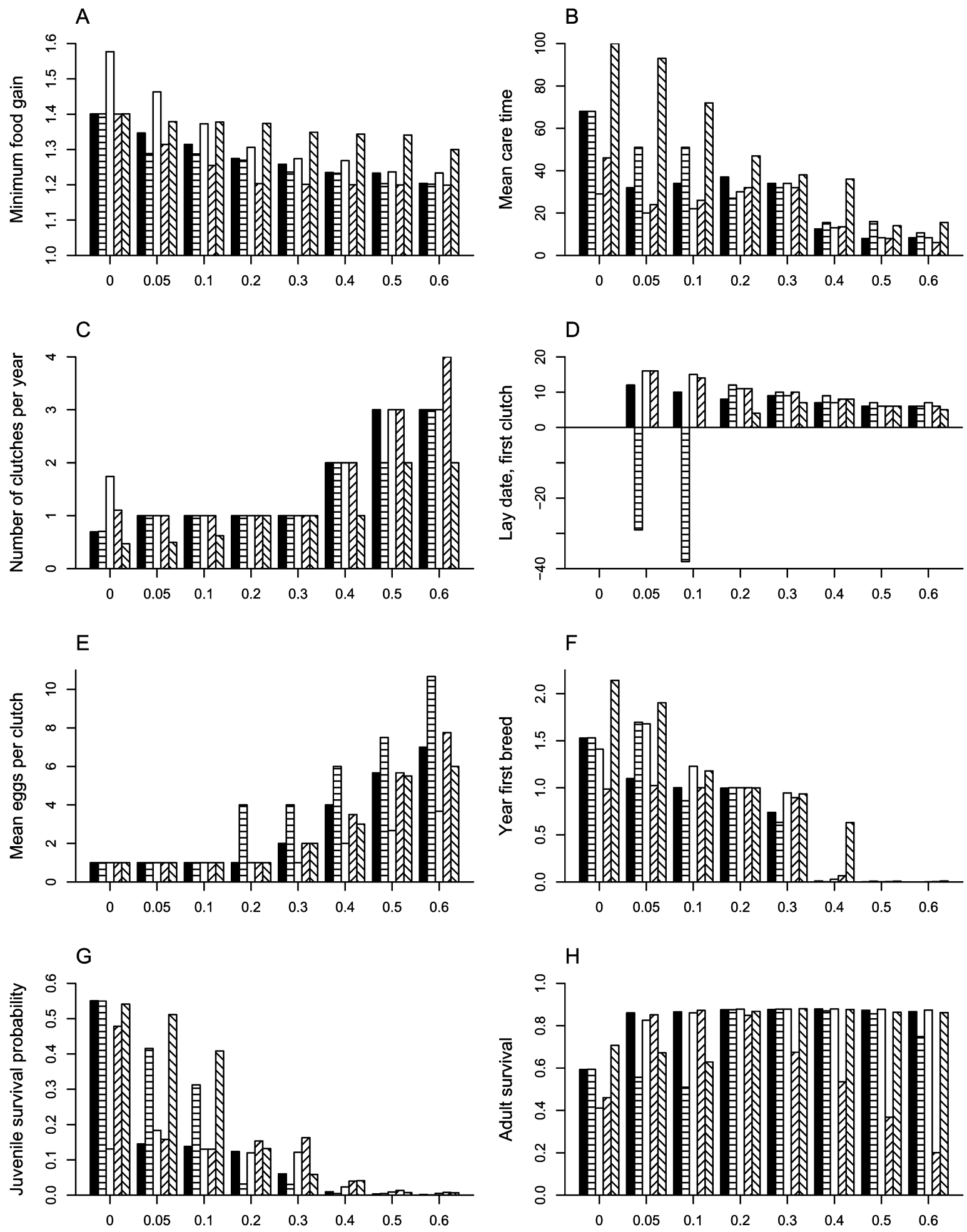

Seasonality, $\varepsilon_{F}$ 
at high seasonality. Age at first breeding tends to be a little higher.

Patterns of juvenile and adult mortality are similar in the two cases. In the predation-risk version, almost all juvenile mortality is the result of starvation in the middle of winter. Most adult mortality is the result of predation. At low $\varepsilon_{\mathrm{F}}$, the main source of mortality is predation during the first few weeks of breeding (when food is still not abundant). For higher $\varepsilon_{\mathrm{F}}$, there is still some mortality associated with breeding, but the main sources are predation and starvation in winter.

\section{The Effect of Nest Predation}

It is not our intention to explore the possible effects of nest predation systematically in this article. Instead we investigate the effect of nest predation in the simple case where predation risk is independent of clutch size and age of dependent young. To do so we take the predation-risk baseline case and add a constant probability $\mu_{\text {off }}$ that dependent young will die each week. The effect of adding this nest predation is illustrated in figure 4 .

Given that mortality costs increase with increasing effort, theory predicts that the effort invested in current reproduction should increase as the value of the current attempt relative to future reproductive success increases. Making nest predation higher reduces the value of the current breeding attempt but also reduces the value of subsequent breeding attempts. Thus, it is not immediately clear that increased nest predation necessarily reduces the value of the current attempt relative to that of future attempts, and so it predicts a reduced effort per breeding attempt. Note, however, that if nest predation reduced success on each breeding attempt, then this would reduce lifetime reproductive success if all else were held fixed. This is, of course, not possible at a density-dependent equilibrium. In our model, density dependence adjusts the food supply so that food availability increases as nest predation increases (fig. 4A). This means that adults are more likely to survive over winter and indeed have greater annual survival at all levels of seasonality despite having to make repeated breeding attempts before one is successful (fig. 4H). This greater survival changes the balance between current and future success, making future success relatively more important. The result is that reproductive effort per attempt is reduced. This can be seen in both the reduced clutch size (fig. $4 E$ ) and the increased age at first breeding (fig. $4 F$ ) as nest predation increases.

\section{Carryover Effects}

Small birds have limited energy storage capacity relative to their rate of energy expenditure (Stuebe and Ketterson 1982; Lehikoinen 1986). For this reason, our model assumes that a bird balances its energy budget during each week; that is, expenditure of energy equals energy intake from food. In contrast, larger birds could potentially build up reserves in one week and use this energy store in future weeks. Furthermore, in addition to energy reserves, all birds have condition measures, such as feather quality and immune competence, that have long-term effects (McNamara and Houston 2008). We do not attempt to make a realistic model of a specific measure of condition here. Instead, we use a simple schematic model of condition to investigate whether our general conclusions are robust when effects carry over from one week to succeeding weeks.

In general, condition might be expected to deteriorate during prolonged periods of forced hard work and to recover (by moulting, in the case of feather quality) once a bird works less hard. We extend our predation baseline model to incorporate this effect as follows. We assume a condition variable $x$ that lies in the range $0 \leq x \leq 1$. If a bird with condition $x$ at the start of one week expends energy at rate $\gamma$ during the week, its condition at the start of the next week is

$$
x^{\prime}=x+\alpha-\beta \gamma
$$

The computations presented in figure 4 are based on the parameter values $\alpha=0.25$ and $\beta=\alpha / 0.9$. For these parameters, the break-even energy expenditure is $\gamma=0.9$. Thus, a bird that is not caring for young and forages for a proportion $u=0.7$ of the week is just able to maintain

Figure 3: Effect of the seasonality in food in the energy-constraint version. Results are at evolutionary stability. Five cases are shown. The baseline case has parameters given in table 1 and a gain in foraging efficiency shown by the middle curve in figure 1 . The low-need and high-need cases differ from this case only in the amount of food that the parent needs to supply to each dependent young (low need: $F_{\text {off }}=0.25$; high need: $\left.F_{\text {off }}=1.0\right)$. The fast-maturation and slow-maturation cases differ from the baseline in that the efficiency gain is given by the top and bottom curves in figure 1, respectively. $A$, Food availability in midwinter $g(0)$. $B$, Mean care time of all broods. $C$, Mean number of clutches per year. $D$, Lay date of the first clutch to become independent during a year. Note that only those cases in which breeding is entrained are shown. $E$, Mean number of eggs per clutch, averaged over all clutches in one year. $F$, Mean year of first breeding. Here years are counted from the time of independence. The year of first breeding is year 0 if breeding occurs before week 52 in the year of independence, year 1 if first breeding occurs in the following calendar year, and so on. G, Probability that an individual survives from independence to the end of week 5 in the following calendar year. $H$, Probability that a fully experienced bird survives a whole year. 
- predation baseline $\boxminus$ nest 0.1

$\square$ nest 0.2

$\square$ condition 6

$\triangle$ condition 100
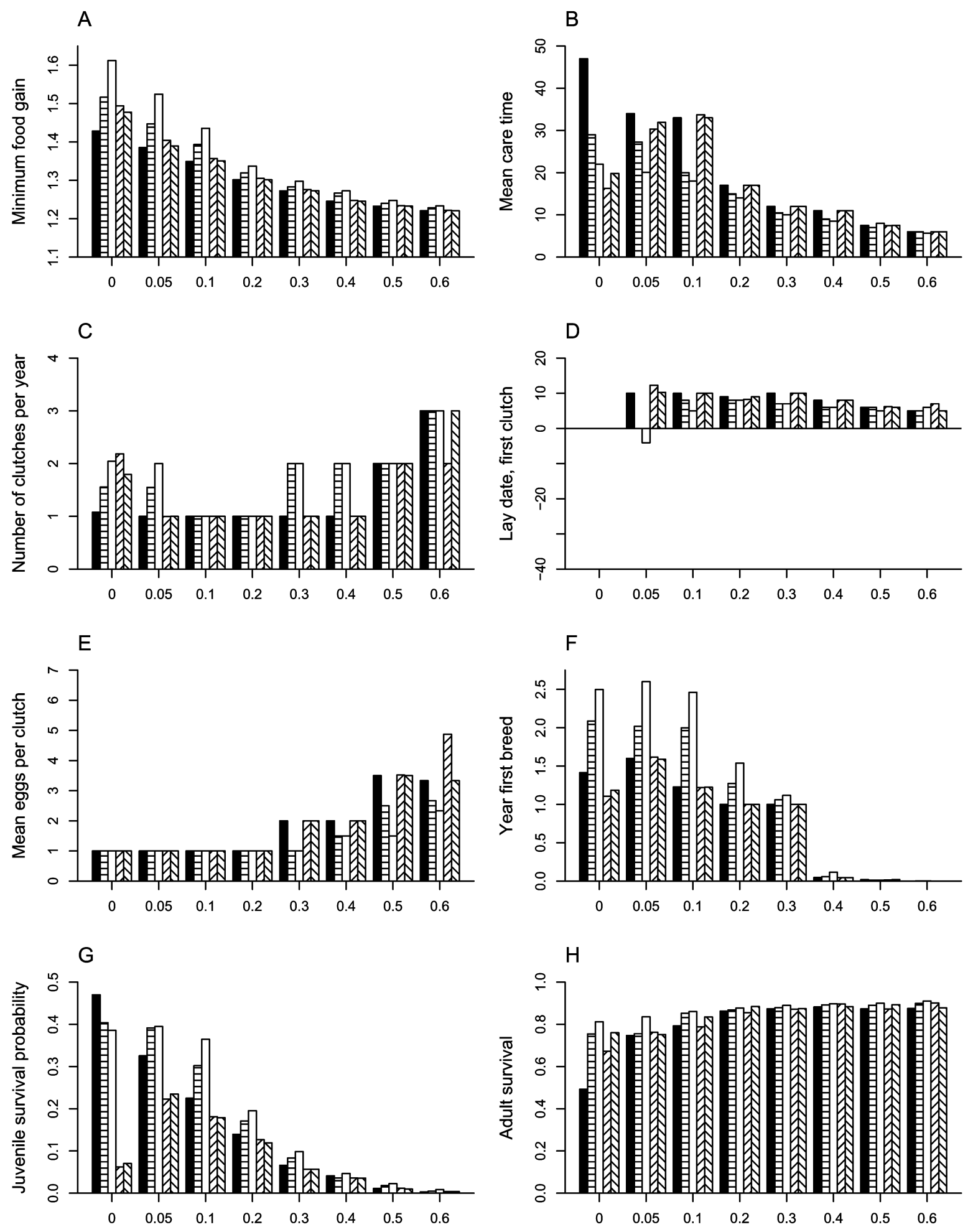

Seasonality, $\varepsilon_{F}$ 
condition. If it works harder, its condition deteriorates; if it works less hard, its condition improves.

We assume that a bird with condition $x$ at the start of the week dies during the week as a results of poor condition with probability

$$
d(x)=(1-x)^{C} .
$$

Figure 4 illustrates the cases $C=6$ and $C=100$. The former case might be appropriate when condition is immune function, as mortality increases rapidly once condition deteriorates sufficiently. In the latter case, the bird dies from poor condition only once condition is very low (essentially 0 ). Thus, this case might be appropriate when condition represents a reserve, such as energy or nutrients. In both cases, at evolutionary stability there are significant carryover effects from week to week; for example, condition may deteriorate progressively during care of young and take several weeks to recover afterward. Despite this, the general effects found in the predation baseline case persist in both of the illustrated cases of carryover (fig. 4).

\section{Robustness to Other Model Assumptions}

Density Dependence. We have assumed that density dependence acts through the parameter $A$. This parameter can be regarded as decreasing with some measure of average annual population density. In appendix D, we instead consider density dependence to be acting locally in time, with food availability in a week being a function of population density that week.

Shape of the Food Availability Function. We have taken the environmental food availability to be a sinusoidal function. This dependence on time of year is probably not realistic at high latitudes, since winters tend to be long while summers are short. Appendix $\mathrm{E}$ in the online edition of the American Naturalist considers the case in which the environmental food availability behaves in this way.

Both the above modifications change the dependence of the food supply on time of year so that it is no longer sinusoidal (fig. D1 in the online edition of the American Naturalist). However, as can be seen from figure D2 in the online edition of the American Naturalist, the qualitative effects of seasonality on the various measures we are concerned with are robust to the change in both the form of density dependence and the environmental food supply.

\section{Seasonality in Temperature}

In appendix $\mathrm{F}$ in the online edition of the American Naturalist, we investigate whether trends shown in figures 3 and 4 can also be obtained by seasonal variation in the resting metabolic expenditure. In particular, we assume that temperature variation is greater at high latitudes, resulting in high resting metabolic expenditure in winter at these latitudes. As can be seen from figure F1, in the examples considered, varying expenditure in this way produces rather weak effects that enhance the effect of the variation in food supply.

\section{Discussion}

Despite the importance of seasonality, relatively little work has been done on its effects on the evolution of life histories. Boyce (1979) develops a relatively simple model, from which he concludes that a strategy with a small clutch size and low demand for resources could be favored when seasonality is low. Boyce's model does not explicitly incorporate clutch size or predation. It also does not model the timing of reproduction and hence cannot make predictions about phenology. Griebeler and Böhning-Gaese (2004) model Ashmole's hypothesis. Their model differs from ours in several respects. They find the optimal clutch size by evolutionary simulations. Because clutch size is the only decision they consider, like Boyce they are unable to make predictions about the timing of breeding, the duration of care, or the age of first reproduction. In addition, the effect of birth date on reproductive value is not included, birds are constrained to produce exactly one clutch per year, and the model of seasonality is schematic. In contrast, we have developed a model that makes predictions about a whole range of life-history variables, including clutch size, number of broods, the time of year at which breeding occurs, and the associated levels of juvenile and adult mortality. Versions of our model incorporate explicit predation and a condition variable. Although we have investigated avian life histories, our approach is completely general and could be used to analyze examples of latitudinal trends in other groups, such as mammals (Lord

Figure 4: Effect of seasonality in food in the predation-risk version. Results are at evolutionary stability. Five cases are shown. The predation baseline case differs from the energy constraint baseline as indicated in table 1. Other cases differ from the predation baseline case as follows. The cases nest 0.1 and nest 0.2 include nest predation ( $\mu_{\text {off }}=0.1$ and 0.2 , respectively). The condition 6 and condition 100 cases are for the model with a condition variable and refer to the cases $C=6$ and 100, respectively (eq. [3]). In these cases, $\alpha=0.25$ and $\beta=\alpha / 0.9$ (eq. [2]). For an explanation of panels, see figure 3 . 
1960; Spencer and Steinhof 1968; Cockburn et al. 1983; Temte 1993) and fish (Leggett and Carscadden 1978; Fleming and Gross 1990; Johnston and Leggett 2002; Heibo et al. 2005). Of course, the details of the models would have to be changed to make them appropriate. For example, environmental temperature is likely to be very important in fish (e.g., Garvey and Marschall 2003; Charnov and Gillooly 2004).

In their model of annual routines of avian moult, Barta et al. (2006) found that the number of breeding attempts per year increased as the environment became more seasonal. They noted that this could be viewed as being in broad agreement with data on latitudinal trends in reproductive effort but pointed out that their model did not allow birds to choose their clutch size. We have presented and analyzed the first self-consistent model of the effect of latitude on avian life history. In this model, the effects of density dependence are crucial. Because of density dependence, it is not the absolute level of food that is important but the maximum level over the year relative to the minimum level, and, as we have argued, an increase in seasonality must reduce midwinter food availability and increase midsummer availability. This effect of seasonality on food availability is responsible for the main effects predicted by our model.

Our model successfully predicts the observed trends in clutch size, duration of parental care, and age at first breeding. The predicted trends are robust. We do not, however, predict the trend in life span (table 2)-as we discuss below, this could be related to the absence of a state variable that represents the long-term effects of hard work. The trend in the number of clutches per year predicted by our model is opposite to the trend observed in the data (Böhning-Gaese et al. 2000). There is, however, a problem in comparing the model's prediction to the data. The results in table 2 are for cases with no nest predation, whereas the data are based on nest predation, which may differ with latitude (Stutchbury and Morton 2001).

Tropical birds generally display a slow pace of life, with lower metabolic rates, lower growth rates, and higher longevities than many temperate-zone counterparts (e.g., Ricklefs 2000b; Stutchbury and Morton 2001; Ricklefs and Wikelski 2002). If such effects are to be understood, a more complex model is required. The evolution of metabolic rate will depend on the negative effects of energy expenditure. As McNamara and Houston (2008) point out, hard work (i.e., a high rate of energy expenditure) has a variety of negative effects that act on different timescales. In this article, we have included a state variable that represents relatively short-term effects. To find the optimal metabolic rate for a given environment, it would be necessary to include a state variable that represents the longterm effects. To model growth, we could include a state
Table 2: Comparison of tropical and temperate birds

\begin{tabular}{|c|c|c|c|}
\hline Feature & Tropical & Temperate & Data \\
\hline$\overline{\text { Clutch size }}$ & 1 & 3.5 & Yes \\
\hline Clutches per year & 1 & 2 & $\mathrm{No}^{\mathrm{a}}$ \\
\hline Mean care time (weeks) ${ }^{\mathrm{b}}$ & 33 & 7.5 & Yes \\
\hline Juvenile survival & .225 & .011 & Unclear $^{\mathrm{a}}$ \\
\hline Year of first breeding & 1.228 & .019 & Yes \\
\hline Adult survival & .793 & .873 & No \\
\hline
\end{tabular}

Note: The comparison is based on the assumption that these regions differ only in terms of seasonality. The first column gives the feature of interest. The second and third columns give predictions from the model for the predation baseline (tropical $\varepsilon_{\mathrm{F}}=0.1$, temperate $\varepsilon_{\mathrm{F}}=0.5$ ). The final column states whether the trend in the predictions is also seen in the data.

${ }^{a}$ Model predictions based on case with no regional differences in nest predation.

${ }^{\mathrm{b}}$ Includes time to build nest and lay eggs.

variable representing physiological maturity in our model. Such a variable would typically mean that newly independent offspring were not physiologically capable of reproduction. Further work could also introduce a state variable to represent the state of repair of the body (see Kirkwood and Rose 1991; Abrams and Ludwig 1995; Kirkwood 1997, 2002; Mangel 2001). We could then consider the evolution of life span and senescence.

Although we compare our results to data from tropical and temperate birds, we are not claiming or assuming that latitudinal trends always exists. What we have shown is that if sites differ only in the seasonality of food, then many of the differences observed between tropical and temperate birds can be predicted by the effect of seasonality. In some cases, however, tropical and temperate regions may differ in several factors, so that no simple trend can be seen. Furthermore, it is possible to find tropical areas that are seasonal. Our model predicts that if all else is equal, a seasonal environment should produce a life history typical of a temperate bird species. It is interesting in this context to note that the green-rumped parrotlet Forpus passerinus inhabits a tropical but seasonal environment close to the equator and has a large clutch size (Beissinger and Waltman 1991) and relatively low adult survival rates (Sandercock et al. 2000).

A fundamental aspect of our model is the interaction between population size and food availability. The importance of this dependence can be seen in the case of increased nest predation. As nest predation increases, density dependence adjusts the food supply so that food availability increases (fig. $4 A$ ). As a result, adults are more likely to survive the winter and have higher annual survival at all levels of seasonality. Because adult survival is higher, future reproductive success becomes relatively more important, and so reproductive effort per breeding attempt is reduced. This example reinforces the view of Barta et 
al. (2006) that it is difficult to attribute an effect to food or predation in isolation.

We have not attempted a complete analysis of nest predation. Future work could allow this source of mortality to depend on clutch size and perhaps also on the age of the brood: dependent young might be in the nest for the first few weeks (subject to one level of predation) but then be out of nest and following the parents, who are still supplying them with food (subject to another level of predation).

We have analyzed a relatively simple model that allows us to isolate the effects of seasonal environments. The basic version of the model does not have a state variable to represent energy reserves; energy balance is the basic constraint on behavior. Models based on energy balance have been used to investigate the ability of parent birds to raise their young (Houston et al. 1996) and the effects of hyenas on wild dogs (Gorman et al. 1998). In contrast to these models, we have explored the implications of energy balance for annual routines in the presence of densitydependent effects. Modifying the basic model to allow foraging to incur a predation risk did not have a strong effect, and the qualitative trends were not changed. These trends did not change when a state variable with carryover effects that lasted a few weeks was added. This shows that energetic arguments of the sort proposed by Lack (1947) and Ashmole (1963) and elaborated by Ricklefs (1980) are sufficient to explain most of the latitudinal trends; see table 2 for details.

\section{Acknowledgments}

We thank S. R. Beissinger and an anonymous reviewer for helpful comments on a previous version of this article. J.M.M. acknowledges support from a Leverhulme Fellowship. Z.B. was supported by a Biotechnology and Biological Sciences Research Council grant to J.M.M. and A.I.H. and by the Hungarian Scientific Research Fund (OTKA; NF 61143, T046661).

\section{Literature Cited}

Abrams, P. A., and D. Ludwig. 1995. Optimality theory, Gompertz' law, and the disposable soma theory of senescence. Evolution 49: 1055-1066.

Ashmole, N. P. 1963. The regulation of numbers of tropical oceanic birds. Ibis 103b:458-473.

Barta, Z., A. I. Houston, J. M. McNamara, R. K. Welham, A. Hedenstrom, T. P. Weber, and O. Fero. 2006. Annual routines of nonmigratory birds: optimal moult strategies. Oikos 112:580-593.

Beissinger, S. R., and J. R. Waltman. 1991. Extraordinary clutch size and hatching asynchrony of a Neotropical parrot. Auk 108:863871.

Beissinger, S. R., M. I. Cook, and W. J. Arendt. 2005. The shelf life of bird eggs: testing egg viability using a tropical climate gradient. Ecology 86:2164-2175.

Böhning-Gaese, K., B. Halbe, N. Lemoine, and R. Oberrath. 2000. Factors influencing the clutch size, number of broods and annual fecundity of North American and European land birds. Evolutionary Ecology Research 2:823-839.

Boyce, M. S. 1979. Seasonality and patterns of natural selection for life histories. American Naturalist 114:569-583.

Bryant, D. M., and C. J. Hails. 1983. Energetics and growth patterns of three tropical bird species. Auk 100:425-439.

Cardillo, M. 2002. The life-history basis of latitudinal diversity gradients: how do species traits vary from the poles to the equator? Journal of Animal Ecology 71:79-87.

Caswell, H. 2001. Matrix population models: construction, analysis, and interpretation. Sinauer, Sunderland, MA.

Charnov, E. L. 1986. Life-history evolution in a recruitment population: why are adult mortality rates constant? Oikos 47:129-134. . 1993. Life history invariants. Oxford University Press, Oxford.

Charnov, E. L., and J. F. Gillooly. 2004. Size and temperature in the evolution of fish life histories. Integrative and Comparative Biology 44:494-497.

Clark, C. W., and M. Mangel. 2000. Dynamic state variable models in ecology. Oxford University Press, Oxford.

Cockburn, A., A. K. Lee, and R. W. Martin. 1983. Macrogeographic variation in litter size in Antechinus (Marsupialia, Dasyuridae). Evolution 37:86-95.

Cody, M. L. 1966. A general theory of clutch size. Evolution 20:174184

Cook, M. I., S. R. Beissinger, G. A. Toranzos, R. A. Rodriguez, and W. J. Arendt. 2003. Trans-shell infection by pathogenic microorganisms reduces the shelf life of non-incubated bird's eggs: a constraint on the onset of incubation? Proceedings of the Royal Society B: Biological Sciences 270:2233-2240.

- 2005. Microbial infection affects egg viability and incubation behavior in a tropical passerine. Behavioral Ecology 16:30-36.

Cooper, C. B., W. M. Hochachka, G. Butcher, and A. A. Dhondt. 2005. Seasonal and latitudinal trends in clutch size: thermal constraints during laying and incubation. Ecology 86:2018-2031.

Dunn, P. O., K. J. Thusius, K. Kimber, and D. W. Winkler. 2000. Geographic and ecological variation in clutch size of tree swallows. Auk 117:215-221.

Fleming, I. A., and M. R. Gross. 1990. Latitudinal clines: a trade-off between egg number and size in Pacific salmon. Ecology 71:1-11.

Garvey, J. E., and E. A. Marschall. 2003. Understanding latitudinal trends in fish body size through models of optimal seasonal energy allocation. Canadian Journal of Fisheries and Aquatic Sciences 60: 938-948.

Ghalambor, C. K., and T. E. Martin. 2001. Fecundity-survival tradeoffs and parental risk-taking in birds. Science 292:494-497.

Gorman, M. L., M. G. Mills, J. P. Raath, and J. R. Speakman. 1998. High hunting costs make African wild dogs vulnerable to kleptoparasitism by hyaenas. Nature 391:479-481.

Griebeler, E. M., and K. Böhning-Gaese. 2004. Evolution of clutch size along latitudinal gradients: revisiting Ashmole's hypothesis. Evolutionary Ecology Research 6:679-694.

Heibo, E., C. Magnhagen, and L. A. Vollestad. 2005. Latitudinal variation in life-history traits in Eurasian perch. Ecology 86:33773386.

Houston, A. I., and J. M. McNamara. 1993. A theoretical investigation 
of the fat reserves and mortality levels of small birds in winter. Ornis Scandinavica 24:205-219.

1999. Models of adaptive behaviour. Cambridge University Press, Cambridge.

Houston, A. I., W. A. Thompson, and A. J. Gaston. 1996. The use of a time and energy budget model of a parent bird to investigate limits to fledging mass in the thick-billed murre. Functional Ecology 10:432-439.

Johnston, J. P., W. J. Peach, R. D. Gregory, and S. A. White. 1997. Survival rates of tropical and temperate passerines: a Trinidadian perspective. American Naturalist 150:771-789.

Johnston, T. A., and W. C. Leggett. 2002. Maternal and environmental gradients in the egg size of an iteroparous fish. Ecology 83:17771791.

Karr, J. R., J. D. Nichols, M. K. Klimkiewicz, and J. D. Brawn. 1990. Survival rates of birds of tropical and temperate forests: will the dogma survive? American Naturalist 136:277-291.

Kirkwood, T. B. L. 1997. The origins of human ageing. Philosophical Transactions of the Royal Society B: Biological Sciences 352:17651772 .

- 2002. Evolution of ageing. Mechanisms of Ageing and Development 123:737-745.

Kirkwood, T. B. L., and M. R. Rose. 1991. Evolution of senescence: late survival sacrificed for reproduction. Philosophical Transactions of the Royal Society B: Biological Sciences 332:15-24.

Klaassen, M. 1994. Growth and energetics of tern chicks from temperate and polar environments. Auk 111:525-544.

Klaassen, M., and R. Drent. 1991. An analysis of hatchling resting metabolism: in search of ecological correlates that explain deviations from allometric relations. Condor 93:612-629.

Koenig, W. D. 1984. Geographic variation in clutch size in the northern flicker (Colaptes auratus): support for Ashmole's hypothesis. Auk 101:698-706.

1986. Geographic ecology of clutch size variation in North American woodpeckers. Condor 88:499-504.

König, S., and E. Gwinner. 1995. Frequency and timing of successive broods in captive African and European stonechats Saxicola torquata axillaris and S. t. rubicola. Journal of Avian Biology 26:247254.

Lack, D. 1947. The significance of clutch-size. I. Intraspecific variations. Ibis 89:302-352.

Leggett, W. C., and J. E. Carscadden. 1978. Latitudinal variation in reproductive characteristics of American shad (Alosa sapidissima): evidence for population specific life-history strategies in fish. Journal of the Fisheries Research Board of Canada 35:1469-1478.

Lehikoinen, E. 1986. Winter ecology of passerines: significance of weight and size. Reports from the Department of Biology 14. University of Turku, Turku.

Lepage, D., and P. Lloyd. 2004. Avian clutch size in relation to rainfall seasonality and stochasticity along an aridity gradient across South Africa. Ostrich 75:259-268.

Lord, R. D. J. 1960. Litter size and latitude in North American mammals. American Midland Naturalist 64:488-499.

Mangel, M. 2001. Complex adaptive systems, aging and longevity. Journal of Theoretical Biology 213:559-571.

Martin, T. E. 1996. Life history evolution in tropical and south temperate birds: what do we really know? Journal of Avian Biology 27:263-272.

- 2002. A new view of avian life-history evolution tested on an incubation paradox. Proceedings of the Royal Society B: Biological Sciences 269:309-316.

Martin, T. E., P. R. Martin, C. R. Olson, B. J. Heidinger, and J. J. Fontaine. 2000. Parental care and clutch sizes in North and South American birds. Science 287:1482-1485.

Martin, T. E., R. D. Bassar, S. K. Bassar, J. J. Fontaine, P. Lloyd, H. A. Mathewson, A. M. Niklison, and A. Chalfoun. 2006. Life-history and ecological correlates of geographic variation in egg and clutch mass among passerine species. Evolution 60:390-398.

McNamara, J. M. 1991. Optimal life histories: a generalisation of the Perron-Frobenius theorem. Theoretical Population Biology 40: 230-245.

McNamara, J. M., and A. I. Houston. 1987. Starvation and predation as factors limiting population size. Ecology Letters 68:1515-1519.

-2008. Optimal annual routines: behaviour in the context of physiology and ecology. Philosophical Transactions of the Royal Society B: Biological Sciences 363:301-319.

McNamara, J. M., R. K. Welham, A. I. Houston, S. Daan, and J. M. Tinbergen. 2004. The effects of background mortality on optimal reproduction in a seasonal environment. Theoretical Population Biology 65:361-372.

Metz, J. A. J., R. M. Nisbet, and S. A. H. Geritz. 1992. How should we define fitness for general ecological scenarios? Trends in Ecology \& Evolution 7:198-202.

Møller, A. P. 2007. Senescence in relation to latitude and migration in birds. Journal of Evolutionary Biology 20:750-757.

Moreau, R. E. 1944. Clutch-size: a comparative study, with special reference to African birds. Ibis 86:286-347.

Orians, G. H., and N. E. Pearson. 1979. On the theory of central place foraging. Pages 154-177 in D. J. Horn, R. D. Mitchell, and G. R. Stairs, eds. Analysis of ecological systems. Ohio State University Press, Columbus.

Peach, W. J., D. B. Hanmer, and T. B. Oatley. 2001. Do southern African songbirds live longer than their European counterparts? Oikos 93:235-249.

Reid, J. M., P. Monaghan, and G. D. Ruxton. 2000. Resource allocation between reproductive phases: the importance of thermal conditions in determining the cost of incubation. Proceedings of the Royal Society B: Biological Sciences 267:37-41.

Ricklefs, R. E. 1976. Growth rates of birds in the humid New World tropics. Ibis 118:179-207.

- 1977a. A note on the evolution of clutch size in altricial birds. Pages 193-214 in B. Stonehouse and C. Perrins, eds. Evolutionary ecology. Macmillan, London.

- 1977b. On the evolution of reproductive strategies in birds: reproductive effort. American Naturalist 111:453-478.

- 1980. Geographical variation in clutch size among passerine birds: Ashmole's hypothesis. Auk 97:38-49.

. 2000a. Density dependence, evolutionary optimization, and the diversification of avian life histories. Condor 102:9-22.

- 2000b. Intrinsic aging-related mortality in birds. Journal of Avian Biology 31:103-111.

Ricklefs, R. E., and M. Wikelski. 2002. The physiology/life-history nexus. Trends in Ecology \& Evolution 17:462-468.

Robinson, W. D., T. R. Robinson, S. K. Robinson, and J. D. Brawn. 2000. Nesting success of understory forest birds in lowland Panama. Journal of Avian Biology 31:151-164.

Russell, E. M. 2000. Avian life histories: is extended parental care the southern secret? Emu 100:377-399.

Russell, E. M., Y. Yom-Tov, and E. Geffen. 2004. Extended parental 
care and delayed dispersal: northern, tropical, and southern passerines compared. Behavioral Ecology 15:831-838.

Sandercock, B. K., S. R. Beissinger, S. H. Stoleson, R. R. Melland, and C. R. Hughes. 2000. Survival rates of a Neotropical parrot: implications for latitudinal comparisons of avian demography. Ecology 81:1351-1370.

Sanz, J. J. 1998. Effects of geographic location and habitat on breeding parameters of great tits. Auk 115:1034-1051.

Schaefer, H. C., G. W. Eshiamwata, F. B. Munyekenye, and K. Böhning-Gaese. 2004. Life-history of two African Sylvia warblers: low annual fecundity and long post-fledging care. Ibis 146:427437.

Skutch, A. F. 1976. Parent birds and their young. University of Texas Press, Austin.

Spencer, A. W., and H. W. Steinhof. 1968. An explanation of geographic variation in litter size. Journal of Mammalogy 49:281-286.

Stuebe, M. M., and E. D. Ketterson. 1982. A study of fasting in tree sparrows (Spizella arborea) and dark-eyed juncos (Junco hyemalis): ecological implications. Auk 99:299-308.

Stutchbury, B. J. M., and E. S. Morton. 2001. Behavioral ecology of tropical birds. Academic Press, San Diego, CA.

Styrsky, J. N., J. D. Brawn, and S. K. Robinson. 2005. Juvenile mortality increases with clutch size in a Neotropical bird. Ecology 86: $3238-3244$.

Sutherland, W. J., A. Grafen, and P. H. Harvey. 1986. Life history correlations and demography. Nature 320:88.
Temte, J. L. 1993. Latitudinal variation in the birth timing of captive California sea lions and other captive North Pacific pinnipeds. Fishery Bulletin 91:710-717.

Tieleman, B. I., J. B. Williams, R. E. Ricklefs, and K. C. Klasing. 2005. Constitutive innate immunity is a component of the paceof-life syndrome in tropical birds. Proceedings of the Royal Society B: Biological Sciences 272:1715-1720.

Weathers, W. W. 1979. Climatic adaptation in avian standard metabolic rate. Oecologia (Berlin) 42:81-89.

1997. Energetics and thermoregulation by small passerines of the humid, lowland tropics. Auk 114:341-353.

Weathers, W. W., and K. A. Sullivan. 1989. Juvenile foraging proficiency, parental effort, and avian reproductive success. Ecological Monographs 59:223-246.

Wiersma, P., A. Muñoz-Garcia, A. Walker, and J. B. Williams. 2007. Tropical birds have a slow pace of life. Proceedings of the National Academy of Sciences of the USA 104:9340-9345.

Wikelski, M., L. Spinney, W. Schelsky, A. Scheuerlein, and E. Gwinner. 2003. Slow pace of life in tropical sedentary birds: a commongarden experiment on four stonechat populations from different latitudes. Proceedings of the Royal Society B: Biological Sciences 270:2383-2388.

Young, B. E. 1994. Geographic and seasonal patterns of clutch-size variation in house wrens. Auk 111:545-555.

Associate Editor: Peter D. Taylor Editor: Michael C. Whitlock 